Supplement of Saf. Nucl. Waste Disposal, 1, 311-311, 2021

https://doi.org/10.5194/sand-1-311-2021-supplement

(c) Author(s) 2021. CC BY 4.0 License.

Supplement of

\title{
Does gentle regulation have a chance?
}

\section{Ulrich Smeddinck}

Correspondence to: Ulrich Smeddinck (ulrich.smeddinck@kit.edu)

The copyright of individual parts of the supplement might differ from the article licence. 


\section{Is there a chance for gentle regulation?}

Ressources of Conflict-Resolution in the Procedure of Siting a Repository for High-Level Radioactive Waste apl. Prof. Dr. Ulrich Smeddinck, KIT/ITAS, Universität Halle-Wittenberg 


\section{Thesis}

- Only if embedded in a comprehensive context of gentle regulation the Repository Siting Act can be implemented successfully. 


\section{What is gentle regulation?}

formats for reducing conflict

- the opposite of top-down regulation

(issue and enforcement of law which is authority-driven and rigorous)

- "gentle" self- and social technologies, characterized by voluntary participation, personal bonds, the unforced force of rational argument or economic incentives (Ulrich Bröckling)

- "Nonetheless, state has to be gentle and open, because it does not exist for its own sake. It is only (...) a means to an end (...), to the development of the single human beings it consists of" (Martin Hochhuth)

- emphasis on communication and discourse 
Forms of gentle regulation in the procedure of siting a repository for high-level radioactive waste

within the Repository Siting Act:

national advisory board

- participation representative

- development of public participation, esp. informal public participation

compensation and socio-economic development

unrelated to the Repository Siting Act:

- political communication and storytelling

- restorative justice, historical analyses, storytelling circle, etc. 


\section{Factors of success}

- enlargement of public participation and reduction of rules providing for the filing of lawsuits within the Repository Siting Act

- invitation to informal activities of public participation which are not legally required

( 5 par. 3 s. 2: The participants can resort to further forms of participation that reach beyond the minimum standard demanded by law.)

attendance and organization of events involving the public

more debate and explanation! 


\title{
Thank you!
}

\author{
ulrich.smeddinck@kit.edu
}

- Form further reading: Smeddinck, Ulrich,

Sanfte Regulierung: Ressourcen der Konfliktlösung im Standortauswahlverfahren für ein Endlager,

Deutsches Verwaltungsblatt (DVBI) 2019, 744-751 
1. EINFÜHRUNG 


\section{Einführung}

- Lorem ipsum dolor sit amet, consetetur sadipscing elitr, sed diam nonumy eirmod

- At vero eos et accusam et justo duo dolores et ea rebum. Stet clita kasd gubergren

- No sea takimata sanctus est Lorem ipsum dolor sit amet.

- Lorem ipsum dolor sit amet, consetetur sadipscing elitr, sed diam nonumy eirmod tempor invidunt 


\section{THEORETISCHER HINTERGRUND}




\section{Theoretischer Hintergrund}

Subline: Arial 20pt bold

- Hier kann Text oder auch ein Bild platziert werden

- ...

a ...

a...
Subline: Arial 20pt bold

Lorem ipsum dolor sit amet, consetetur sadipscing elitr, sed diam nonumy eirmod tempor invidunt ut labore et dolore magna aliquyam erat, sed diam voluptua.

At vero eos et accusam et justo duo dolores et ea rebum. Stet clita kasd gubergren. 


\section{Lorem ipsum dolor sit amet}

- Lorem ipsum dolor sit amet, consetetur sadipscing elitr, sed diam nonumy eirmod

- At vero eos et accusam et justo duo dolores et ea rebum. Stet clita kasd gubergren

- No sea takimata sanctus est Lorem ipsum dolor sit amet.

- Lorem ipsum dolor sit amet, consetetur sadipscing elitr, sed diam nonumy eirmod tempor invidunt 


\section{EMPIRISCHE ERGEBNISSE}




\section{Musterüberschrift: Arial 24pt bold}

Subline: Arial 20pt bold

- Hier kann Text oder auch ein Bild platziert werden

- ...

- ...

a ...

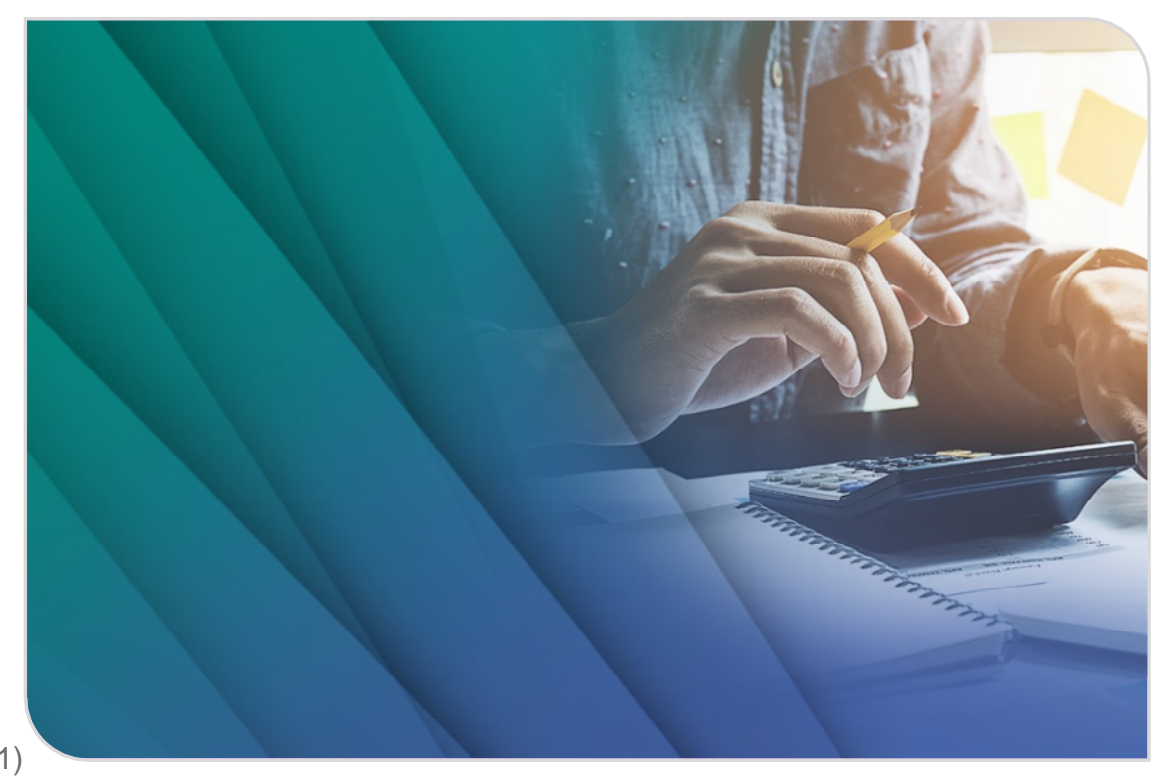




\section{FAZIT}


Musterüberschrift: Arial 24pt bold

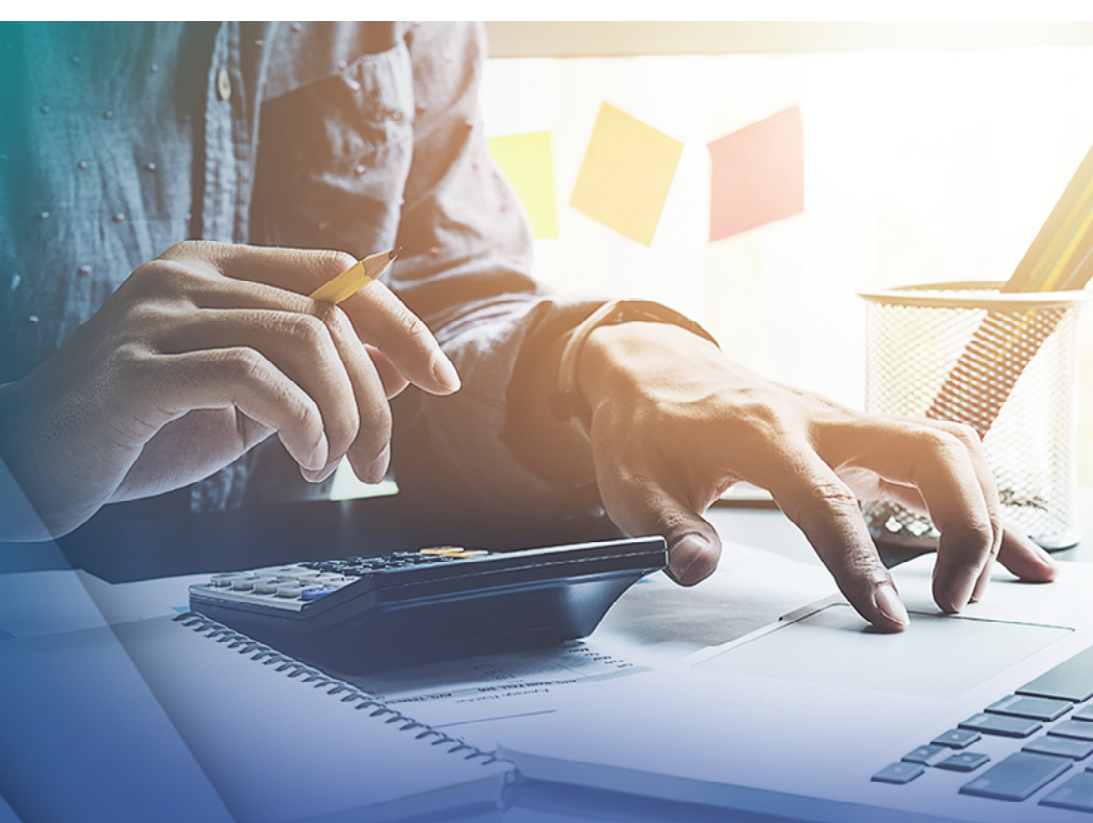




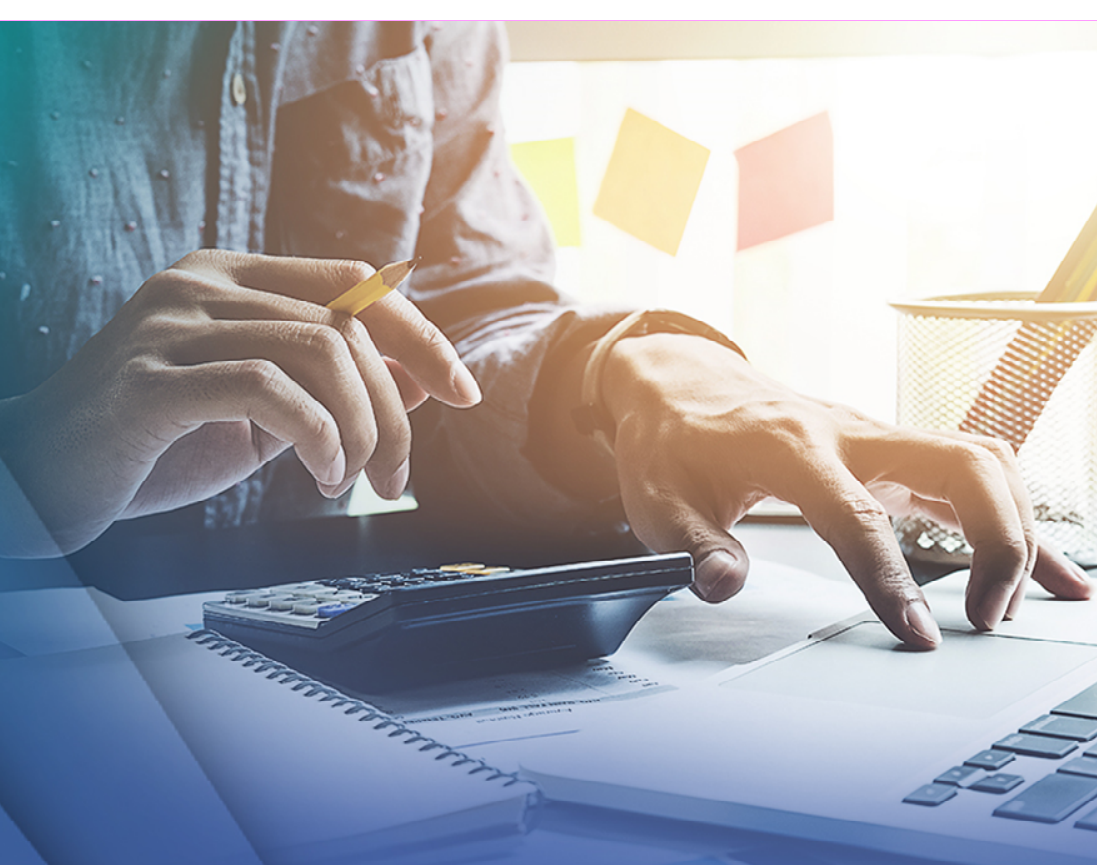




\section{Vielen Dank für Ihre Aufmerksamkeit!}

Gefördert durch:

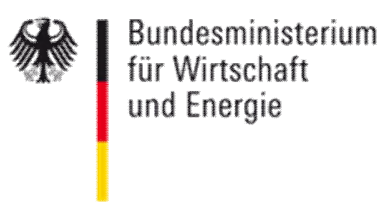

aufgrund eines Beschlusses des Deutschen Bundestages
Gefördert im

Niedersächsischen Vorab der Volkswagenstiftung

$\therefore:$ VolkswagenStiftung

$\because \div$

2. Niedersächsisches Ministerium

für Wissenschaft und Kultur

Förderkennzeichen: 02E11849A-J 


\section{Literatur}

Lorem ipsum dolor sit amet, consetetur sadipscing elitr, sed diam nonumy eirmod tempor invidunt

Lorem ipsum dolor sit amet, consetetur sadipscing elitr, sed diam nonumy eirmod tempor invidunt

Lorem ipsum dolor sit amet, consetetur sadipscing elitr, sed diam nonumy eirmod tempor invidunt

Lorem ipsum dolor sit amet, consetetur sadipscing elitr, sed diam nonumy eirmod tempor invidunt

Lorem ipsum dolor sit amet, consetetur sadipscing elitr, sed diam nonumy eirmod tempor invidunt 


\section{Bildquellen}

(1) https://pixabay.com/de/

(2) https://www.pexels.com/de-de/

(3) https://unsplash.com/

(4) Lorem ipsum dolor sit amet, consetetur sadipscing elitr, sed diam nonumy

(5) Lorem ipsum dolor sit amet, consetetur sadipscing elitr, sed diam 